\title{
EFEITO DA DIREÇÃO DA TRANSFERÊNCIA INTERLATERAL NO APRENDIZADO DE TAREFA DE SEQUÊNCIA DE TOQUES DE DEDOS*
}

\author{
GRAD. PAULO CÉSAR ANDREAN \\ Licenciado em Educação Física, Universidade Estadual de Londrina (Londrina - Paraná - Brasil) \\ E-mail: paulocean@hotmail.com \\ MS. BRUNO SECCO FAQUIN \\ Mestre em Educação Física, Universidade Estadual de Londrina (Londrina - Paraná - Brasil) \\ E-mail: brunopoti@hotmail.com \\ DRA. JULIANA BAYEUX DASCAL \\ Doutora em Educação Física, Universidade Estadual de Londrina (Londrina - Paraná - Brasil) \\ E-mail: jbdascal@yahoo.com.br \\ DR. VICTOR HUGO ALVES OKAZAKI \\ Doutor em Educação Física, Universidade Estadual de Londrina - \\ Programa de Educação Tutorial da Educação Física (PET-EF) (Londrina - Paraná - Brasil) \\ E-mail: vhaokazaki@gmail.com
}

\begin{abstract}
RESUMO
O presente estudo analisou o efeito da direção da transferência interlateral no aprendizado de tarefa de toques de dedos. Vinte participantes destros ( $18-30$ anos) foram divididos em 2 grupos: prática com a mão direita (GD) e com a mão esquerda (GE). Os grupos foram avaliados em fases de pré-teste, pós-teste e retenção. A tarefa consistiu na realização do maior número de toques alternados entre o polegar e os demais dedos, durante 10 segundos, em sequência pré-estabelecida. O GE apresentou maior número de toques de dedos com a mão esquerda no pós-teste $(Z=2,2 ; P=0,028)$ comparado ao pré-teste. $O$ GD aumentou o número de toques de dedos do pré-teste para a retenção para a mão direita $(Z=2,4$; $P=0,017)$ e esquerda $(Z=2,6 ; P=0,009)$, indicando o aprendizado e a transferência interlateral. A transferência interlateral no sentido da mão direita para esquerda foi explicada pelo princípio da treinabilidade.
\end{abstract}

PALAVRAS-CHAVE: Transferência interlateral; habilidade motora; toque de dedos; aprendizagem motora.

I. $\quad$ presente estudo contou com apoio financeiro da CAPES (modalidade de bolsa de mestrado concedida ao segundo autor) e do MEC/SeSu (modalidade de bolsa de tutor do Programa de Educação Tutorial da Educação Física concedida ao último autor). 
A transferência interlateral diz respeito à capacidade de transferir parte do conhecimento ou de elementos aprendidos, em função da prática de uma habilidade motora realizada com um lado do corpo, para o outro lado que não realizou a prática (MAGILL, 200 I ; TEIXEIRA, 2006). Desta forma, o aprendiz não necessita recomeçar do início o processo de aprendizagem quando se deparar com uma situação em que irá realizar a mesma tarefa com o membro contra-lateral que ainda não praticou. Esta capacidade de transferir conhecimento ou elementos de uma habilidade motora praticada pode ser verificada no desempenho de uma habilidade motora para outras habilidades motoras, e do ambiente da prática para outros ambientes (MAGILL, 200 I; SCHMIDT; WRISBERG, 200 I). De modo geral, o fenômeno da transferência interlateral pode ser explicado de três formas, a saber: aprendizagem de elementos cognitivos, mecanismos de controle motor e compartilhamento de redes neurais.

A explicação baseada na aprendizagem dos elementos cognitivos postula que o que é transferido é a informação importante relacionada com o que se pretende atingir no desempenho da habilidade motora. Como resultado da prática com um membro, é adquirida informação cognitiva importante, a qual é disponibilizada quando a habilidade motora é desempenhada com o membro contra-lateral. Baseada na teoria dos elementos idênticos de Thorndike (19|4), a vertente cognitiva considera todos os elementos da habilidade motora relacionados com "o que fazer" como sendo primariamente informação cognitiva sobre o desempenho dessa habilidade.

Outra forma de explicar a transferência interlateral está baseada nos mecanismos de controle motor, os quais podem apresentar duas vias: uma incorpora os programas motores generalizados e a outra se refere às questões da ativação neuromuscular. Em relação aos programas motores generalizados, estes operam como mecanismo de controle, pela especificação das características do movimento relacionadas com o tempo e com o espaço, numa relação integrada de percepção e ação (VASCONCELOS, 2006). Por meio do desenvolvimento suficiente de um programa motor generalizado para um dos membros, parece ser possível atingir um nível razoável de desempenho com o outro membro.

Quanto à ativação neuromuscular (HICKS; GUALTIERI; SCHROEDER, 1983), tem sido sugerido que pelo menos alguma transferência interlateral é mediada pela comunicação inter-hemisférica, ao nível do corpo caloso, relativamente a informações sobre os componentes motores da tarefa. Esta mediação pode ser demonstrada por meio de registros eletromiográficos detectados no membro inativo durante a prática realizada com o membro oposto (DAVIS, 1942). Como seria de 
esperar, a explicação sobre o fenômeno da transferência interlateral decorre de uma interação constante das duas teorias, sendo resultado de fatores cognitivos, motores e neuromusculares. Estas teorias auxiliaram no entendimento das razões pelas quais a experiência motora adquirida com a prática com um membro do corpo influencia no aprendizado do membro homólogo contra-lateral.

De acordo com Wrisberg ( 1993), o achado mais significante sobre transferência interlateral é que essa transferência é assimétrica entre os membros, ou seja, existem diferenças em termos de direção da transferência. Em situações práticas em que a preferência manual não é restringida, observa-se uma tendência de utilização inicial do lado preferido, para posteriormente praticar com o lado não-preferido. Este comportamento pode estar relacionado à maior segurança e conforto gerados no desempenho com o membro preferido (FAQUIN, 20I2). Entretanto, os resultados dos estudos são contraditórios em relação à melhor direção da transferência. Tem sido demonstrado que a melhor direção da transferência pode ocorrer do membro preferido para o não-preferido (BYRD; GIBSON; GLESON, 1986; WANG; SAINBUG, 2004; INUI, 2005) ou do membro não-preferido para o preferido (TAYLOR; HEILMAN, I980; KUMAR; MANDAL, 2005) e, ainda, que a direção da transferência entre membros depende de parâmetros específicos da tarefa, como força e timing (TEIXEIRA, 2000; 2006). Como exemplo, Thut et al. ( 1996) utilizaram uma tarefa de desenho de padrões geométricos e observaram que, para a medida de tempo de movimento, a direção da transferência mais efetiva foi do membro preferido para o membro não-preferido, enquanto que para a medida de precisão espacial, a melhor direção ocorreu no sentido contrário.

Diferentes hipóteses explicativas são encontradas sobre os níveis de ativação hemisférica gerados pelo membro praticado, preferido ou não-preferido, e pelas características das tarefas. Taylor e Heilman (1980) afirmam que a prática com a mão não-preferida leva a uma ativação cerebral bihemisférica, facilitando assim a transferência para o membro preferido. Assim, a prática com a mão preferida (direita) levaria à maior ativação do hemisfério esquerdo, resultando em menor grau de transferência para o membro não-preferido. Segundo Teixeira (2006), determinados componentes da tarefa, como o controle de força e timing coincidente, são controlados com a mesma proficiência por ambos os hemisférios cerebrais, o que gera uma transferência simétrica entre os membros nesses parâmetros.

Apesar do conhecimento da existência da transferência interlateral durante a aprendizagem, ainda não é conhecido o efeito da direção dessa transferência. Como a preferência lateral tende a atribuir maior experiência motora para um dos lados do corpo (lado preferido), magnitudes diferenciadas de transferência interlateral ocorreriam quando a prática de uma habilidade motora assimétrica fosse realizada. 
Por exemplo, a prática inicial com o membro não-preferido poderia proporcionar maior transferência para o lado preferido. Isto, pois o lado preferido já possui maior experiência motora, sendo necessária apenas a transferência de alguns elementos cognitivo-motores para auxiliar no aprendizado. Ao passo que a prática no sentido preferido para não-preferido apresentaria menor transferência interlateral, uma vez que o lado não-preferido possuiria menor experiência para aproveitar os elementos transferidos com a prática realizada com o lado contra-lateral homólogo. Entretanto, ainda não é conhecido em que sentido (preferido para não-preferido ou vice-versa) ocorre maior transferência de aprendizado. Por conseguinte, são necessários mais estudos que investiguem o efeito da transferência interlateral no aprendizado de habilidades motoras assimétricas.

Considerando os pontos discutidos anteriormente, o presente estudo analisou o efeito da direção de transferência interlateral sobre o aprendizado em tarefa de sequência de toques de dedos. $O$ presente estudo tem potencial para auxiliar no entendimento de qual sentido ocorre maior magnitude de transferência de aprendizado. Tal conhecimento pode auxiliar professores de educação física e técnicos para a escolha da ordem do lado que irá realizar a prática primeiro, durante o aprendizado de uma habilidade motora assimétrica.

\section{MÉTODO}

\section{PARTICIPANTES}

A amostra foi constituída por 20 indivíduos, homens e mulheres, com idade entre 18 e 30 anos. Os participantes foram divididos aleatoriamente em dois grupos de 10 participantes cada, conforme descrição a seguir: (a) grupo direita (GD) realizou a prática da tarefa com a mão direita, e (b) grupo esquerda (GE) realizou a prática da tarefa com a mão esquerda. Apenas sujeitos que apresentaram preferência manual direita, indicada pelo inventário de Edimburgo (OLDFIELD, I97I), participaram do estudo. Antes do início dos testes todos os participantes assinaram um termo de consentimento livre e esclarecido. Os procedimentos do estudo foram aprovados pelo Comitê de Ética em Pesquisa da universidade local (parecer n $217 / 10$, CAAE $n^{\circ}$ 0 199.0.268.000- I0, folha de rosto $n^{\circ} 36857$ I).

\section{INSTRUMENTOS E TAREFA}

Foi utilizada como tarefa para o estudo, a realização de movimentos de toques alternados entre o polegar e os demais dedos, na seguinte sequência: indicador, anelar, médio e mínimo. Cada tentativa correspondeu a efetuar o maior número de 
toques possíveis em 10 segundos, respeitando a sequência mencionada. $\bigcirc$ desempenho nesta tarefa foi medido por meio de uma luva com sensores, mensurando-se a quantidade de toques, desde o primeiro toque entre o dedo indicador e o polegar, até o último toque entre o dedo mínimo e o polegar, durante 10 segundos. Para a tentativa ser considerada válida não foi permitido mais que dois erros por tentativa. Neste caso, o erro correspondeu a errar a sequência proposta para o toque de dedos, ou seja, indicador, anelar, médio e mínimo. A luva utilizada para a mensuração dos números de toques interdígitos foi a Eletronic Fingers' Adaptor for Timing Tasks (v. I .2; OKAZAKI, 2007), que foi acoplada a um adaptador analógico-digital (Analog-to-Digital Adaptor for Laboratory Tasks v. I .0; OKAZAKI, 2007). Este adaptador analógico-digital permitiu integrar a luva ao software Finger Sequence Task (v. I .2; OKAZAKI, 2007), que forneceu a variável dependente de estudo.

\section{PROCEDIMENTOS EXPERIMENTAIS}

Os participantes foram instruídos que não poderiam cometer erro na sequência de toques dos dedos na tarefa, sendo explicado o que seria o erro. 0 participante apenas iniciou ou parou a tentativa ao comando de quem coletava os dados. Antes de iniciar a coleta de dados, foi permitido aos participantes realizarem duas tentativas de familiarização com tarefa, uma com cada mão. A posição inicial para desempenhar a tarefa foi com o participante sentado, com os pés apoiados no chão e a mão voltada para frente, o cotovelo desta mão apoiado sobre a mesa e olhando para frente, não sendo permitido que o participante olhasse para a mão a fim de evitar o feedback visual (FIGURA I). A posição inicial de forma incorreta não foi considerada como erro, pois enquanto o participante não corrigisse a posição não era iniciada a tentativa.

a)

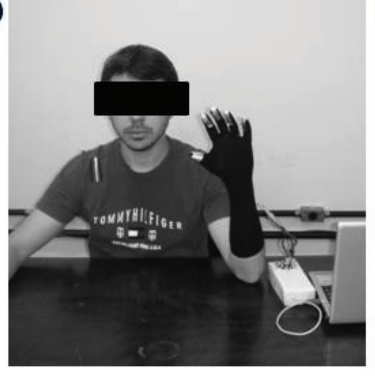

b)

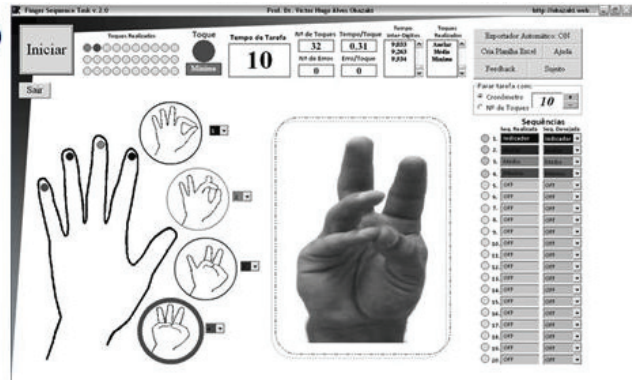

Figura I . a) Representação esquemática da realização da tarefa de toque de dedos com a luva com sensores e b) software específico de análise. 
Na sequência, foram realizadas as seguintes fases de avaliação, a saber: (I) pré-teste com 3 tentativas realizadas com cada mão; (2) aquisição com 3 sessões em dias diferentes ( 24 horas de intervalo entre as sessões), sendo que em cada sessão foram realizados 3 blocos de 20 tentativas (GD praticou com a mão direita e GE praticou com a mão esquerda), com intervalo de 10 segundos entre as tentativas e I minuto entre os blocos; (3) pós-teste com 3 tentativas realizadas com cada mão; (4) retenção, após 72 horas com 3 tentativas também realizadas com cada mão; e (5) transferência inter-tarefa, realizada no mesmo dia da retenção, com 3 tentativas também realizadas com cada mão. Nesta fase a tentativa correspondeu à sequência que já vinha sendo feita, porém na ordem inversa, ou seja, mínimo, médio, anelar e indicador.

\section{VARIÁVEIS DE ESTUDO}

As variáveis independentes do estudo foram: os grupos (prática com a mão esquerda e prática com a mão direita) e as fases de teste (Pré-teste, Pós-teste, Retenção e Transferência). A variável dependente do estudo foi o número de toques (NT). Como variável de controle foi utilizado o erro nas sequências dos toques de dedos.

\section{ANÁLISE ESTATIISTICA}

A análise descritiva dos dados foi apresentada por meio de medianas e intervalos interquartis. Para a análise inferencial nas comparações entre os grupos (GD e GE) foi utilizado o teste de Mann Whitney-U. Para as comparações intra-grupos foi utilizado o teste de ANOVA de Friedman seguido, quando necessário, pelo teste de Wilcoxon pareado. A significância adotada foi de 5\% (P $\leq 0,05)$.

\section{RESULTADOS}

Foi verificado, por meio do teste de Mann Whitney- $U$, melhor desempenho na mão esquerda do grupo que praticou com a mão esquerda (GE), em comparação a mão esquerda do grupo que praticou com a mão direita (GD), durante o pós-teste $(Z=-2,9 ; P=0,004)$. A Figura 2 apresenta os resultados em medianas e intervalos interquartis. 


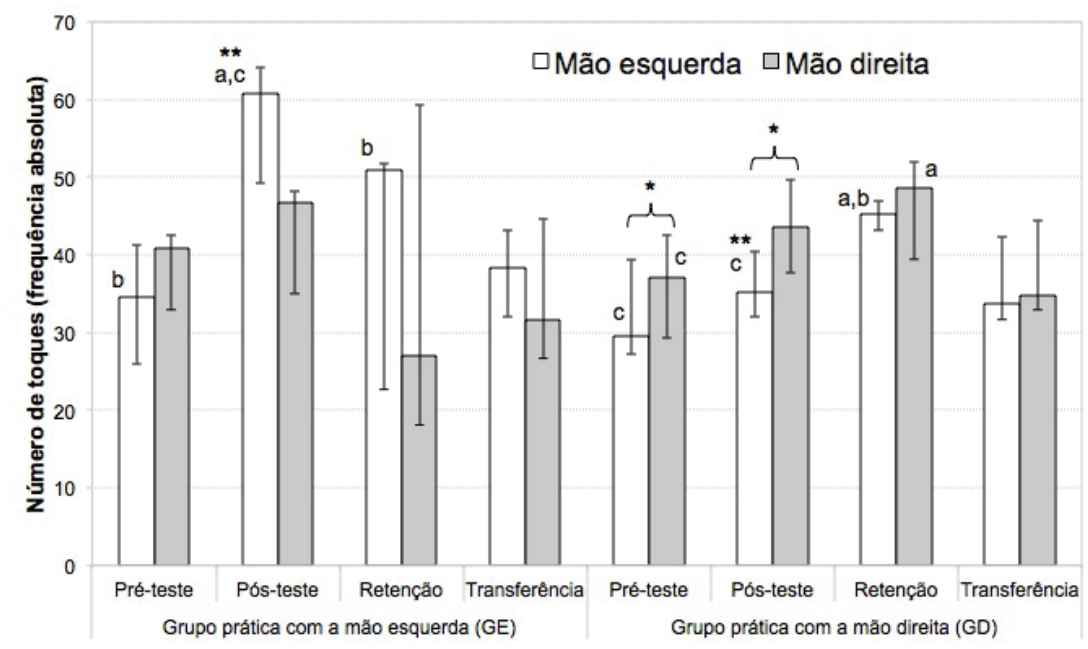

Figura 2. Números de toques de dedos (mediana, $1^{\circ}$ quartil e $3^{\circ}$ quartil) dos grupos GD e GE, em função das fases de teste (pré-teste, pós-teste, retenção e transferência).

Legenda: diferença $(P<0,05)$ em comparação: "às mãos direita e esquerda; " "aos grupos GD e GE durante a mesma fase de teste; a ao pré-teste no mesmo grupo; bao pós-teste no mesmo grupo; e, cà retenção no mesmo grupo.

teste de ANOVA de Friedman demonstrou diferença entre as condições do grupo $G D\left(X^{2} f_{(N=10, g l=7)}=24,2 ; P=0,00 I\right)$. Foi verificado maior número de toques de dedos com a mão direita, em comparação à mão esquerda, no grupo que praticou com a mão direita, durante o pré-teste $(Z=2,4 ; P=0,017)$ e o pós-teste $(Z=2,5 ; P=0,0 \mid 3)$. $\bigcirc$ pré-teste realizado com a mão direita (GD) apresentou menor número de toques de dedos em comparação à retenção na mesma mão $(Z=2,4 ; P=0,0 \mid 7)$. A retenção realizada com a mão esquerda (GD) apresentou melhor desempenho em relação ao pré-teste $(Z=2,6 ; P=0,009)$ e ao pós-teste $(Z=2,7 ; P=0,007)$.

Para o grupo GE, o teste de ANOVA de Friedman também demonstrou diferença entre as condições $\left(X^{2} f_{(N=\mid 0, g=7)}=|8 ; P=0,0| \mid\right)$. Comparando as fases do $\mathrm{GE}$, houve maior número de toques de dedos com a mão esquerda no pós-teste, em comparação ao pré-teste $(Z=2,2 ; P=0,028)$ e à retenção $(Z=2,7 ; P=0,008)$.

\section{DISCUSSÃO}

presente estudo analisou o efeito da direção de transferência interlateral no aprendizado em tarefa de sequência de toques de dedos. Foi levantada a 
hipótese de que a prática inicial com o membro não-preferido proporcionaria maior transferência para o lado preferido. Uma vez que o lado preferido possui maior experiência motora, deste modo, sendo apenas necessária a transferência de alguns elementos cognitivo-motores para auxiliar no aprendizado do membro homólogo contra-lateral.

O grupo que praticou com a mão esquerda apresentou melhor desempenho no pós-teste realizado com a mesma mão em função da prática. Entretanto, houve uma piora no desempenho da mão esquerda do pós-teste para a retenção. Tais resultados indicam uma melhora expressiva no desempenho inicial, porém sem aprendizado. Esta ausência de aprendizado foi explicada pela quantidade de prática ou pelo tempo necessário para ocorrer a aprendizagem (SINGER; HAUTALA, 1968 apud COSTA, 1991). Segundo Singer e Hautala ( 968 apud COSTA, 199 I), a aprendizagem e a magnitude da transferência interlateral têm relação direta com a quantidade de prática realizada. Faquin et al. (20ll) apontam que deve haver uma quantidade expressiva de aprendizagem e de aperfeiçoamento no desempenho, decorrentes da prática, para que a transferência interlateral ocorra. A maior dificuldade em determinar este tempo necessário, ou quantidade de prática, para a aprendizagem está no fato de que o aprendizado deve levar em consideração fatores, tais como as características dos aprendizes e da habilidade motora em questão (SINGER; HAUTALA, 1968 apud COSTA, 199I). No presente estudo, foi sugerido que a quantidade de prática para a mão esquerda foi insuficiente para garantir o aprendizado e a transferência interlateral.

A prática com a mão esquerda também proporcionou melhor desempenho para esta mão, em comparação à mão direita no pós-teste. Este melhor desempenho da mão esquerda durante o pós-teste proporcionou uma assimetria de desempenho entre as mãos. Porém, esta assimetria não foi mantida para a retenção, em função do desempenho não ter sido estabilizado com a pouca quantidade de prática necessária para esta mão. Foi verificado maior número de toques de dedos com a mão direita para o GD, em comparação à mão esquerda, durante o pré-teste e o pós-teste. Tais resultados demonstraram uma assimetria de desempenho entre as mãos, favorável para a mão direita. Desempenhos superiores com a mão preferida em relação à mão não-preferida em tarefas de toques repetidos também têm sido encontrados em outros estudos de lateralidade (DENCKLA, 1974; INGRAM, 1975; PETERS, 1980, 198I; PROVINS; CUNLIFFE, I972; TODOR; KYPRIE, 1980). Peters (198I) mostrou que essa assimetria de desempenho é devida à modulação superior de força da mão preferida, produzindo circuitos rápidos de contração/relaxamento de grupos musculares antagônicos do punho para produzir movimentos de flexão/extensão de alta frequência. Em tais tarefas, o desempenho com a mão preferida tem mostrado 
não apenas ser mais rápido, mas também mais consistente, mesmo após um período extensivo de treinamento (PETERS, 1981). Apesar desta vantagem verificada para a mão direita, após a prática os desempenhos ficaram simétricos (testes de retenção e transferência). Isso indica que a prática pode equalizar o desempenho entre os lados, mesmo que haja maior preferência lateral por utilizar um determinado lado (PETERS, 1976; PROVINS; GLENCROSS, 1968).

Foi verificado efeito de aprendizagem no grupo de prática com a mão direita. Esta prática com a mão direita também demonstrou melhorar o número de toques de dedos na fase de retenção realizada com a mão esquerda. Tais resultados indicaram a transferência interlateral da mão direita para a esquerda. Tais resultados sugerem que o aprendizado é uma condição sine qua non para que a transferência de aprendizagem ocorra. Os resultados do presente estudo também têm suporte nos estudos de Ammons ( | 958) apud Vasconcelos ( 199 |). Este autor sugere que ocorre maior transferência da mão direita para a esquerda, pois a primeira mão teria mais experiência em tarefas com componentes semelhantes aos praticados. Deste modo, o grau de aprendizagem que ocorreria a fim de se atingir proficiência com a mão direita não seria tão grande quanto o grau exigido para a mão esquerda (AMMONS, 1958 apud VASCONCELOS, 1991). Esta racionalidade é baseada no princípio da treinabilidade, que aponta que 'quanto mais treinado for um indivíduo, menos treinável ele será' e vice-versa. Ou seja, quanto mais experiência um aprendiz possuir, maior será seu desempenho e, consequentemente, menos susceptível ao aperfeiçoamento ele será. A recíproca também seria verdadeira, de modo que, quanto menor for a experiência do aprendiz, mais sensível à prática será o aprendiz para o aperfeiçoamento de seu desempenho. Deste modo, como a mão direita possui maior experiência, esta mão necessitaria de mais prática para apresentar modificação expressiva em seu desempenho. Por outro lado, como a mão esquerda possui menor experiência, esta mão estaria mais susceptível ao efeito da transferência de aprendizado.

\section{CONCLUSÃO}

Os resultados do presente estudo demonstraram transferência interlateral no sentido da mão direita (preferida) para a esquerda (não-preferida). Desta forma, foi refutada a hipótese levantada de que haveria maior magnitude de transferência interlateral no sentido da mão não-preferida para a preferida. A maior transferência interlateral no sentido da mão preferida para não-preferida foi explicada pelo princípio da treinabilidade. Foi sugerida a realização de outros estudos sobre a temática de transferência de aprendizagem interlateral que considerem a utilização de maior tempo de prática e diferentes habilidades motoras para serem aprendidas. 


\section{Effect of interlateral transfer of learning direction in touching finger sequence task}

ABSTRACT: The present study analyzed the effect of interlateral transfer direction while learning a touching finger sequence task. Twenty right-handed participants ( 18 -30 years old) were divided into two groups: practice with the right hand (GD) and left hand (GE). Groups were evaluated during pretest, posttest, and retention phases. The task was performing the higher number of finger touches between tomb and the other fingers, during 10 seconds, in a pre-stablished order. The GE showed higher number of finger touchs with the left hand in posttest $(Z=2,2 ; P=0,028)$ compared to pretest. The $G D$ increased the number of finger touches from pretest to retention for the right hand $(Z=2,4 ; P=0,0 / 7)$ and left hand $(Z=2,6$; $P=0,009)$, indicating learning and interlateral transfer of learning. The interlateral transfer of learning from the right to the left hand was explained by the principle of trainability.

KEYWORDS: Interlateral transfer; motor Skill; touch of fingers; motor Learning.

\section{Efecto de la dirección de la transferencia interlateral de aprendizaje en la} tarea de secuencia de tocar los dedos

RESUMEN: Este estudio examinó el efecto de la dirección de la transferencia interlateral en la tarea de secuencia de tocar los dedos. Veinte participantes diestros (18-30 años) fueron divididos en dos grupos: la práctica con la mano derecha (GD) y la mano izquierda (GE). Los grupos fueron evaluados en el pre-test, post-test y la retención. La tarea era hacer el mayor número de movimientos alternantes entre el pulgar y otros dedos, por 10 segundos, con una secuencia preestablecida. GE realizado mayor número de toques de dedos con la mano izquierda en el post-test $(Z=2.2, P=0,028)$ en comparación con el pre-test. El GD aumentado el número de movimientos del dedo en la retención de la mano derecha ( $Z$ $=2.4, P=0,017)$ e izquierda $(Z=2.6, P=0,009)$, lo que indica el aprendizaje y la transferencia interlateral. La transferencia interlateral hacia la mano derecha a la izquierda se explica por el principio de la treinabilidad.

PALABRAS CLAVE: Transferencia interlateral; habilidad motora; tarea de tocar los dedos; aprendizaje motor.

\section{REFERÊNCIAS}

BYRD, R.; GIBSON, M.; GLEASON, M. H. Bilateral transfer across ages 7 to 17 years. Perceptual and Motor Skills, Missoula, v. 62, p. 87-90, 1986.

COSTA, O. Desporto e qualidade de vida. In: BENTO, J.; MARQUES, A. (Ed.). Actas das Jornadas Cientificas Desporto, Saúde e Bem Estar. Porto: FCDEF, 1991.

DAVIS, R. C. The pattern of muscular action in simple voluntary movements. Journal of Experimental Psychology, Washington, v. 31, p. 437-66, 1942. 
DENCKLA, M. B. Development of motor co-ordination in normal children. Developmental Medicine and Child Neurology, Malden, v. 16, p. 720-742, 1974.

FAQUIN, B. S. et al. Interlateral transference of learning in dart throwing. The FIEP Bulletin, v. 8 I, p. 38I-384, 20II.

FAQUIN, B. S. Efeito da atenção sobre a preferência lateral e o aprendizado em tarefas motoras. 201 2. Dissertação (Mestrado em Educação Física) - Universidade Estadual de Londrina, Londrina, 2012.

HICKS, R. E.; GUALTIERI, C. T.; SCHROEDER, S. R. Cognitive and motor components of bilateral transfer. American Journal of Psychology, Champaign, v. 96, p. 223-228, 1983.

INGRAM, D. Motor asymmetries in young children. Neuropsychologia, Philadelphia, v. I3, p. 95-10, 1975.

INUI, N. Lateralization of bilateral transfer of visuomotor information in right-handers and left-handers. Journal of Motor Behavior, New York, v. 37, p. 275-283, 2005.

KUMAR, S.; MANDAL, M. Bilateral transfer of skill in left- and right-handers. Laterality: asymmetries of body, brain, and cognition, New York, v. I0, p. 345-352, 2005.

MAGILL, R. A. Motor learning: concepts and applications. 6 ${ }^{\text {th }}$. ed. New York: McGraw Hill, 2001 .

OKAZAKI, V. H. A. Analog-to-digital adaptor for laboratory tasks (v. I.0). Hardware utilizado para integração do software Finger Sequence Task (v.l.2) de mensuração de toques de dedos. 2007.

OKAZAKI, V. H. A. Eletronic fingers' adaptor for timing tasks (v. I .2). Software de mensuração da tarefa de toque sequência de dedos. Disponível em: http://okazaki.webs.com. 2007.

OKAZAKI, V. H. A. Finger Sequence Task (v. I .2). Software de análise das variáveis dependentes da tarefa de toque de dedos. Disponível em: http://okazaki.webs.com. 2007.

OLDFIELD, R. C. The assessment and analysis of handedness: the Edinburgh inventory. Neuropychologia, v. 9, p. 97-113, 1971.

PETERS, M. Prolonged practice of a simple motor task by preferred and nonpreferred hands. Perceptual and Motor Skills, Missoula, v. 43, p. 447-450, 1976.

PETERS, M. Handedness: effect of prolonged practice on between hand performance differences. Neuropsychologia, Philadelphia, v. 19, n. 4, p. 587-590, 1981.

PETERS, M. Why the preferred hand taps more quickly than the non-preferred hand: three experiments on handedness. Canadian Journal of Psychology, v. 34, n. I, p. 62-7, 1980.

PROVINS, K. A.; CUNLIFFE, P. The reliability of some motor performance tests of handedness. Neuropsychologia, Philadelphia, v. 10, p. 199-206, 1972. 
PROVINS, K. A.; GLENCROSS, D. J. Handwriting, typewriting and handness. The Quarterly Journal of Experimental Psychology, v. 20, n. 3, p. 282-289, 1968.

SCHMIDT, R. A.; WRISBERG, C.A. Aprendizagem e performance motora. 2. ed. Porto Alegre: Artmed, 200I.

TAYLOR H. G.; HEILMAN K. M. Left-hemisphere motor dominance in righthanders. Cortex, v. I6, p. 587-603, 1980.

TEIXEIRA, L. A. Timing and force components in bilateral transfer of learning. Brain and Cognition, v. 44, p. 455-469, 2000.

TEIXEIRA, L. A. Intermanual transfer of timing control between tasks holding different levels of motor complexity. Laterality, v. I I, p. 43-56, 2006.

THORNDIKE, E. L. Educational psychology. New York: Century, 1914.

THUT, G. et al. Intermanual transfer of proximal and distal motor engrams in humans. Experimental Brain Research, v. 108, p. 321-327, 1996.

TODOR, J. I.; KYPRIE, P. M. Hand differences in the rate and variability of rapid tapping. Journal of Motor Behavior, v. I2, n. I, p. 57-62, 1980.

VASCONCELOS, O. Strength, accuracy and dexterity with respect to sex, handedness and personal activities. In: CONGRESSO ESPANHOLA DE ANTROPOLOGIA BIOLÓGICA, 4., p. 562-569. Bilbao, 1991. Actas... Bilbao, 1991.p. 562-569.

VASCONCELOS, O. Aprendizagem motora, transferência bilateral e preferência manual. Revista Brasileira de Educação Física, São Paulo, v. 20, 37-40, 2006.

WANG, J.; SAINBURG, R. L. Limitations in interlimb transfer of visuomotor rotations. Experimental Brain Research, v. I 55, p. I-8, 2004.

WRISBERG, C. A. Levels of performance skill. In: SINGER, R. N.; MURPHEY, M.; TENNANT, L. K. (Ed.). Handbook of Research on Sport Psychology. New York: MacMillan, 1993.

Recebido em: 7 jan. 2012

Aprovado em: 29 set. 2012

Endereço para correspondência:

Prof. Dr. Victor Hugo Alves Okazaki

Departamento de Educação Física - Universidade Estadual de Londrina Campus Universitário - Rodovia Celso Garcia Cid Km 380 - Caixa Postal: 600 I - CEP: 8605 I-990 - Fone/Fax: (55) (43) 337I-5857 - Londrina - Paraná - Brazil

E-Mail: vhaokazaki@gmail.com

Site: http://okazaki.webs.com \& http://petef.webs.com 